\title{
Measurement by infrared thermography of skin temperature variations in mice undergoing a surgery event
}

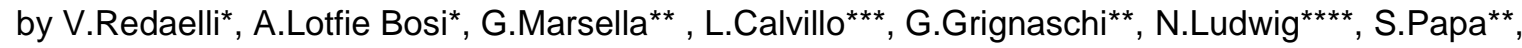 \\ P.Veglianese ${ }^{\star \star}$, I.Vismara $^{\star *}$, S.Rimondo ${ }^{\star \star}$, F.Luzi ${ }^{\star}$ \\ *Università degli Studi di Milano, Dipartimento di Medicina Veterinaria, via G. Celoria, 10, 20133, Milano, Italy \\ ** IRCCS Istituto di Ricerche Farmacologiche Mario Negri, Animal Care Unit, via La Masa, 19, 20156, Milano, \\ Italy \\ *** IRCCS Istituto Auxologico Italiano, Laboratory of Cardiac Arrhythmias on Genetic Base, via Zucchi, 18, \\ 20095, Cusano Milanino, Italy

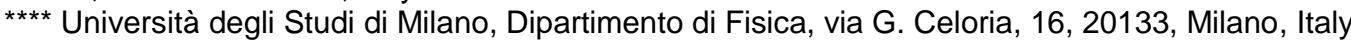

\begin{abstract}
As IRT is potentially a great tool according to the EU laws, a survey was carried out on mice after a surgery event recording skin temperature variations on selected body areas. Measures have been taken the day before the surgery, immediately after, at 1, 7, 14 and 21 days after. A similar trend was found: temperatures increase until the day 7 and then come down reaching values not significatively different from the basal ones at day 21 . This first results are useful to provide reference values for a most common daily use of IRT technique in animal lab facilities.
\end{abstract}

\section{Introduction}

The use of the animal model in preclinical studies is strictly regulated by the EU Directive $63 / 2010$ that has been transposed in Italy into the D.Lvo 26/2014. In the light of this legislation and of the 3Rs principle [1], the use of non-invasive but objective methods to assess the physiological state and health of the subjects has become a mandatory requirement. The thermographic technique has both of these characteristics and has been used for example to investigate the physiological reactions in response to stressful and fear stimuli in rats [2] and rabbits [3].

The consistency and repeatability of this technique in measuring skin temperature was verified, for example, on nude mice [4]. Aim of this study is to investigate skin temperature variations in selected areas after a surgery event.

\section{Materials and methods}

The thermographic survey was divided into three tests, carried out in three different seasons, on $36 \mathrm{C} 57 \mathrm{BI} / \mathrm{n}$ mice after a surgery event of Spinal cord injury. Subjects were male, adult, individually housed in IVC cages with ad libitum access to water and standard rodent feed, in the SPF laboratory at the IRCCS Mario Negri Institute for Pharmacological Research in Milan. Room temperature will be maintained at $21^{\circ} \pm 1^{\circ} \mathrm{C}$ and relative humidity at $50 \% \pm$ $20 \%$. Animals will be kept on a 12/12 light/dark cycle.

The surgery, performed under total anaesthesia according with EU legislation, consists in the compression of the spinal cord at the level of the T13 vertebrae, which results in a lesion to the hind legs, mimicking the human paraplegia. The animals received two different analgesic treatments, whose efficacy was evaluated using various behavioural analyses (Mouse grimace scale, Mouse behaviour analysis, Home cage scan). The fur in the region of interest was cut the day of the surgery.

Animals' skin temperature was recorded the day before the surgery (basal), immediately after the surgery, after 24 hours and at 7,14 and 21 days after surgery, always between 10 a.m. and 13 p.m.. The more interest area were that of the brown adipose tissue (BAT), on the back of the animals, and on the tail. IRT was performed as first, before any other manipulation, when the mouse arrived from the housing area and lay in its cage (TO), and after 30 minutes wen mouse finished the behavioural analyses.

An infrared camera FLIR A65 with a microbolometer sensor of $640 \times 512$ pixels was used. At less 3 images for each subject were stored and analysed with Flir Tools and IRT Analyzer software. The average of the max temperature value on the brown fat was extracted. Non parametric one way tests were performed and di p-value (p) was set at 0,05 . 


\section{Results and discussion}

All skin temperatures recorded after surgery showed a similar trend (Fig. 2-3). The skin temperature in the BAT area drop in the surgery day and then increased and reached the max value at the day 7 , at more than $37^{\circ} \mathrm{C}$, attesting the better thermoregulatory response. During the days after the temperature come down, maybe for the regrowth of the fur that isolates the animals. No significatively differences were found in BAT temperature between the two different analgesic treatments. More, the individual variability in the temperature of the tails was too great in order to obtain some valid statistical results (Fig.4).

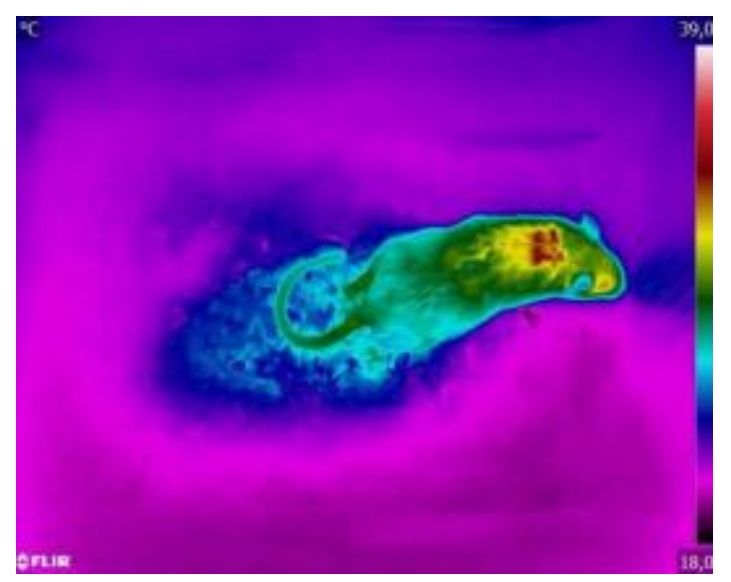

Fig. 1. Example of IRT images recorded. The hottest region $(r e d)$ is the brown adipose tissue.

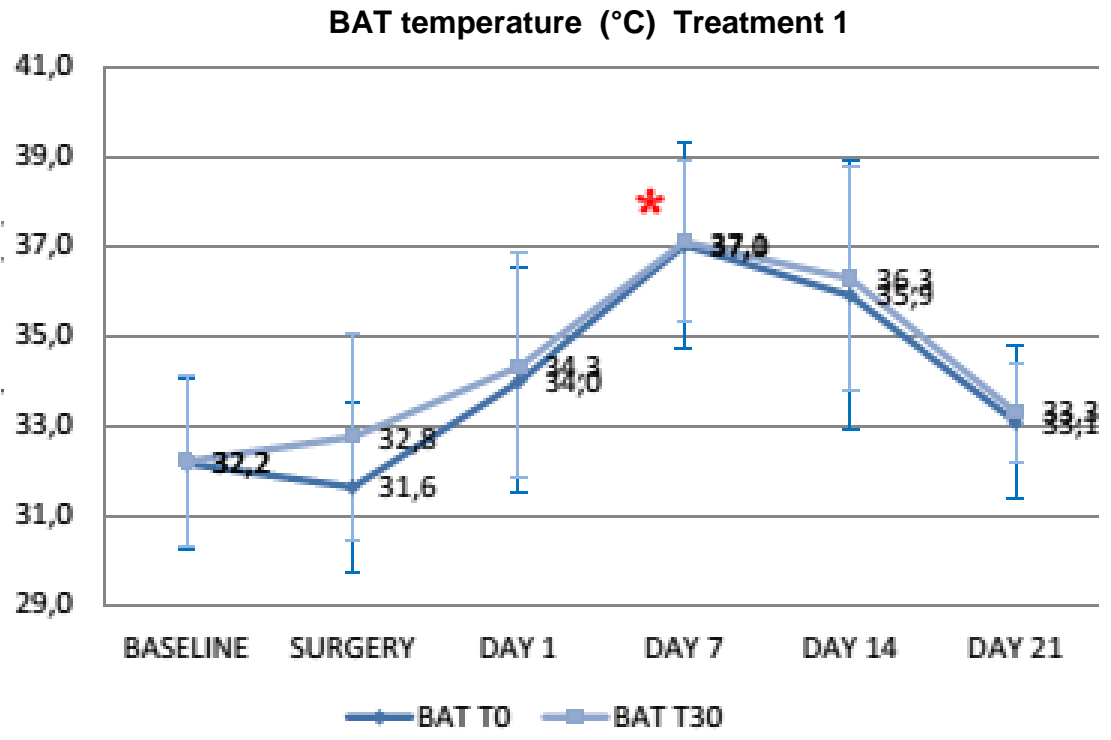

Fig.2. Average temperature of the brown adipose tissue at TO and T30 for mice with analgesic treatment 1 


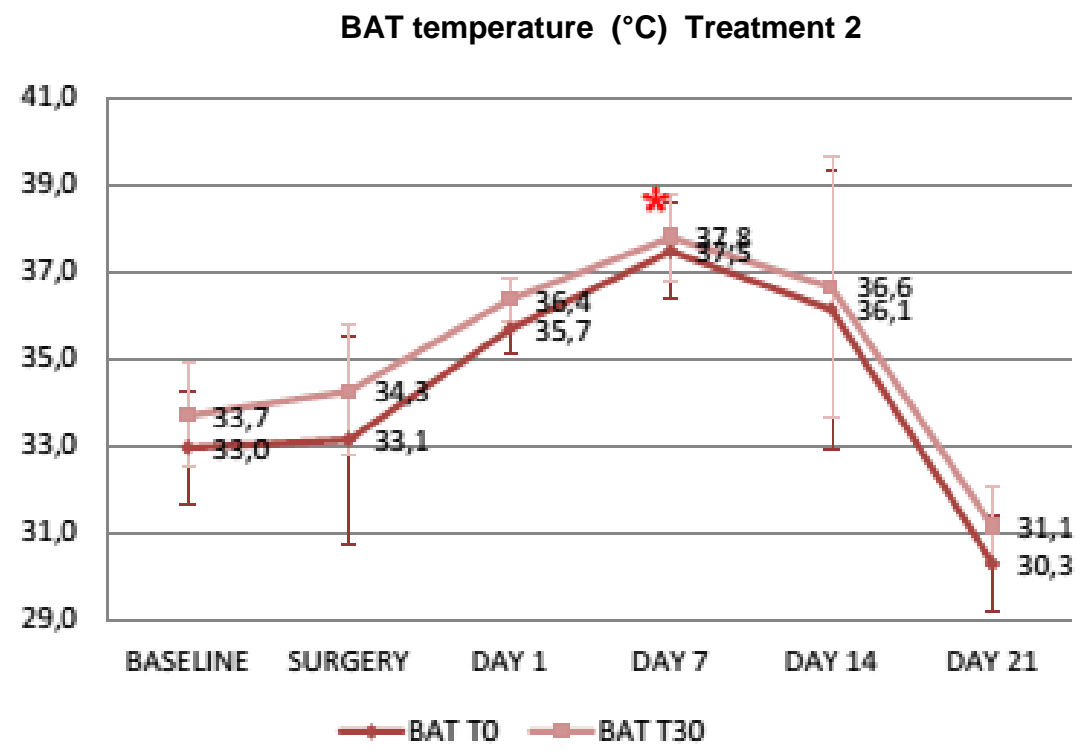

Fig. 3. Averages temperatures of the brown adipose tissue at TO and T30 for mice with analgesic treatment 2

TAIL temperature $\left({ }^{\circ} \mathrm{C}\right)$

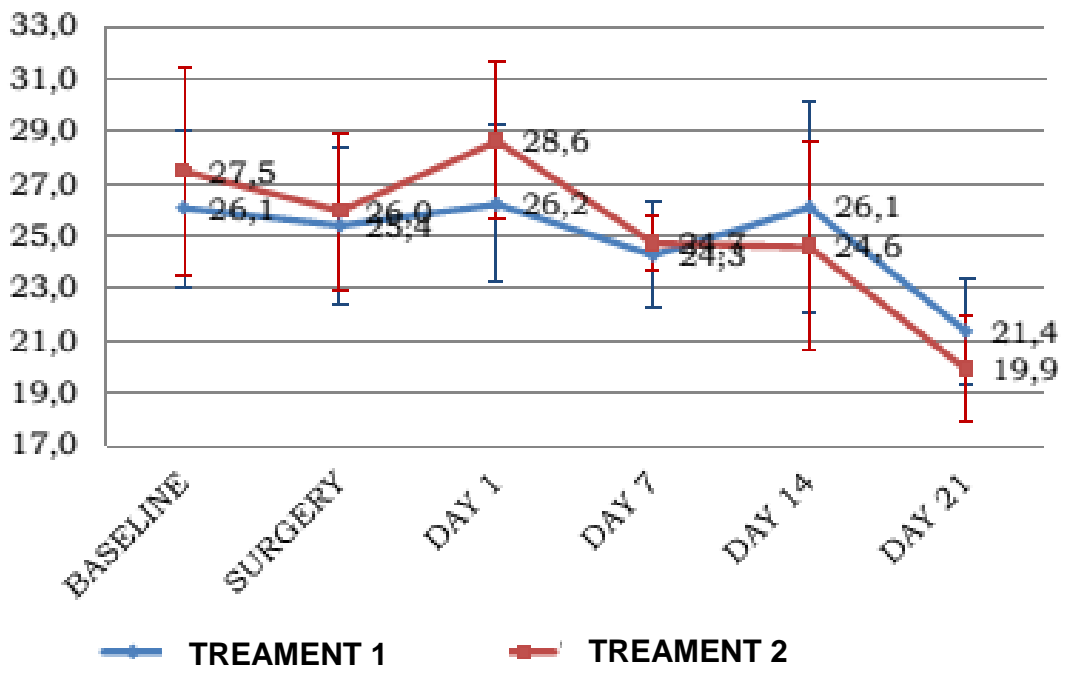

Fig. 4. Average temperatures of the tails at TO 


\section{Conclusions}

All skin temperatures recorded in the region of Brown adipose tissue after surgery showed a similar trend, with an increase from basal values that reach the max at the day 7 and then come down reaching values not significatively different from the basal one. The area with the stronger temperature increase was the brown adipose tissue with an average amount of a bit less than $5{ }^{\circ} \mathrm{C}$.

First results here obtained are useful to better understand physiological and thermoregulatory response of mice subjected to manipulations typical of surgery events and to provide reference values for a most common daily use of IRT technique in animal lab facilities. In fact IRT is potentially a great tool according to the 3R principal.

\section{REFERENCES}

[1] Kolar R, (2006). Animal experimentation. Sci. Eng. Ethics. 2006, Jan; 12.1: 111 -22.

[2] Vianna, D.M.L. and Carrive, P. (2005) Changes in cutaneous and body temperature during and after conditioned fear to context in the rat. Proc. Aust. Neuroscience Soc., 21:2505-2512.

[3] Ludwig N, Gargano M, Luzi F, Carenzi C, Verga M, (2007). Applicability of infrared thermography as a non invasive measurement of stress in rabbits. World Rabbit Science, ISSN 1257-5011, 15(4).

[4] Fornasier M., Redaelli V., Tarantino A., Luzi F., Verga M. (2010). Infrared thermography (IRT) in nude mice: an alternative method for body temperature measurement. Atti Scand FELASA 2010, Helsinki, June 14-17, 2010 